\title{
Signalling function of long wavelength colours during agonistic male-male interactions in the wrasse Coris julis
}

\author{
Christoph Braun $^{1, *}$, Nico K. Michiels ${ }^{2}$, Ulrike E. Siebeck ${ }^{1}$, Dennis Sprenger ${ }^{2}$ \\ ${ }^{1}$ School of Biomedical Sciences, University of Queensland, St. Lucia 4072, Australia \\ ${ }^{2}$ Animal Evolutionary Ecology, Institute for Ecology and Evolution, University of Tübingen, Auf der Morgenstelle 28 E5, \\ 72076 Tübingen, Germany
}

\begin{abstract}
Long wavelength colours $(\lambda>580 \mathrm{~nm})$ often serve as visual signals during inter- and intrasexual interactions in various species of freshwater fish. However, while long wavelength colours are also prevalent in many marine fish, their functional importance remains largely unknown. Here, we demonstrate that the presence of long wavelength colours mediate intraspecific aggressive interactions in the Mediterranean rainbow wrasse Coris julis. By manipulating the relative mixture of red $\left(\lambda_{\max }=628 \mathrm{~nm}\right)$ and blue $\left(\lambda_{\max }=454 \mathrm{~nm}\right)$ light in experimental tanks, we created conditions in which discrimination of long wavelength colours from colours of shorter wavelength was either possible or prevented. We found that resident males were significantly more likely to attack conspecific intruder males when discrimination was enabled compared to conditions where no such discrimination was possible. Aggression from residents was associated with the display of a red $\left(\lambda_{\max }=611 \mathrm{~nm}\right)$ colour patch on the dorsal fin of intruders, but not the size of the putative signal. Our findings suggest that long wavelength colours are an important component of marine visual ecology by mediating agonistic male-male interactions.
\end{abstract}

KEY WORDS: Behaviour · Colour signals $\cdot$ Labrid $\cdot$ Visual ecology

\section{INTRODUCTION}

Colour signals in fish often indicate an individual's physical abilities (Andersson \& Iwasa 1996) and are generally displayed in the context of social interactions, such as territorial fights (Stacey \& Chiszar 1978, Baube 1997, Seehausen et al. 1997, Siebeck 2004), the establishment and enforcement of dominance hierarchies (Bakker \& Sevenster 1983) and female mate choice (Bakker \& Mundwiler 1994). The available light spectrum is dependent on the optical properties of water, with long and short wavelength colours being attenuated the strongest due to absorption and scattering of incident light (Jerlov 1968, Lythgoe 1979). Despite the resulting limitations for short and long wavelength signals in aquatic environments, previous work on various species of fresh- water fish inhabiting shallow waters showed that orange-red colour displays $(\lambda>580 \mathrm{~nm})$ can be naturally and sexually selected signals that are important components of species and mate recognition, respectively (Milinski \& Bakker 1990, Seehausen et al. 1997, Seehausen \& van Alphen 1998, Franck et al. 2003).

The functional importance of long wavelength colours as visual signals in the sea, however, remains largely unknown. This is mainly because the majority of marine fish live in blue-green light dominated waters (Jerlov 1968) and have visual pigments that are most sensitive to that part of the spectrum (Marshall et al. 2003). There is some anecdotal evidence in the scientific literature suggesting a potential signalling function of long wavelength colours $(\lambda>$ $580 \mathrm{~nm}$ ) in marine fish. First, many marine fish spe- 
cies display colour patterns in the long wavelength range (Marshall et al. 2003). Second, some fish, amongst which are also labrids, are documented to possess long wavelength sensitive photoreceptors in the retina in addition to the short and medium wavelength sensitive cones (Barry \& Hawryshyn 1999, Douglas et al. 2000, Marshall et al. 2006, Kondrashev 2008, Michiels et al. 2008), and therefore can be considered to have trichromatic vision. However, it remains unknown whether these receptors contribute to colour vision. Finally, the production of red light in deeper waters, where the long wavelength spectrum from sunlight is absent, has been demonstrated in reef fish and deep sea fish in the form of red biofluorescence (Michiels et al. 2008, Sparks et al. 2014) and red bioluminescence (Douglas et al. 2000), respectively.

We tested for the signalling function of long wavelength colours during aggressive male-male interactions in the haremic, sex-changing Mediterranean rainbow wrasse, Coris julis Linnaeus, 1758. During peak breeding season from April to September, male rainbow wrasses defend breeding territories along rocky shore lines and sea grass meadows from $1 \mathrm{~m}$ to at least $50 \mathrm{~m}$ depth (Lejeune 1987). Following sex change from female to male, individual fish increase growth rates and change colour such that terminal phase males display a conspicuous lateral orange zigzag stripe (henceforth $\mathrm{ZZS}_{\text {; }}$ see Fig. 1a) along each side of the body as well as a prominent red patch on the first few spines of the dorsal fin (henceforth DFP for dorsal fin patch; see Fig. 1a). Behavioural observations from the field suggest a potential signalling function of the dorsal fin, and therefore possibly the DFP, as the fin is frequently displayed during courtship (Duchac et al. 1982) and agonistic male-male interactions (C. Braun unpubl. data) in shallow (1 to $10 \mathrm{~m}$ ) and deeper $(>30 \mathrm{~m})$ waters. We quantified the aggressive response of resident fish towards intruder male conspecifics in 4 different light environments. Specifically, we tested the hypotheses that (1) residents are more likely to attack intruders in the presence versus the absence of red light. Because relative signal size has been demonstrated to influence aggressive interactions in territorial males (Basolo 1990, Pryke et al. 2001, 2002, Benson \& Basolo 2006), we made the general prediction that (2) the likelihood of aggression depends on the relative difference in signal size of the DFP and the ZZS between residents and intruders. Finally, we expected (3) the number of attacks by residents to increase with increasing intensity of red in the light environment.

\section{MATERIALS AND METHODS}

\section{Study location and sampling}

Experiments were conducted at the Station de Recherches Sous-marines et Océanographiques (STARESO), Corsica, France in summer 2010. All specimens $(n=84)$ were collected from different social groups and sites around Punta Revellata $\left(42^{\circ} 34^{\prime} 51^{\prime \prime} \mathrm{N}, 8^{\circ} 43^{\prime} 32^{\prime \prime} \mathrm{E}\right)$, a peninsula near STARESO. Fish were caught with the use of SCUBA gear and a hand net, and placed into 151 buckets with closed lids during the short $(<5 \mathrm{~min})$ boat transport back to STARESO. Following transfer to the laboratory, the total length (TL, from snout to caudal fin) of each fish was measured to the nearest $\mathrm{mm}$ by placing the fish into a transparent Ziploc bag with a small amount of water and gently holding the fish against a ruler taped to the surface of a table. All research and experiments complied with French law, and fish were caught with the permission of STARESO.

\section{Spectrometric measures}

Natural light environment

In order to characterize the natural light environment at different depths at Punta Revellata, we measured spectral radiance using a PR-670 spectroradiometer (Photo Research) in a custom-made underwater housing aimed at a Spectralon 99\% diffuse white standard (Labsphere) fixed at $20 \mathrm{~cm}$ in front of the lens in a $45^{\circ}$ angle, facing south at noon (4 July 2010 ). Spectra taken at depths of 1, 3, 5, 7, 9 and $10 \mathrm{~m}$ were used to calculate the diffuse attenuation coefficient, $\alpha$, of ambient water using the equation:

$$
I=I_{0} \mathrm{e}^{-d_{\alpha}}
$$

where $I_{0}$ and $I$ are the spectral irradiances separated vertically by distance, $d$, in $\mathrm{m}$. Results for different depths were averaged. We used the attenuation coefficient to estimate the amount of light in deeper water.

Field measures of Coris julis

Spectral radiances of the orange ZZS and DFP of a male rainbow wrasse were measured with the spectrometer mentioned above, in the field under natural light conditions. Measurements were made at $23 \mathrm{~m}$ 
depth near Punta Revellata on a sunny day around noon (see Fig. A1b in the Appendix). A male C. julis was anaesthetized with a mixture of clove oil, ethanol and seawater and gently pushed onto its side on a mat black slate oriented towards the water surface. The ZZS and DFP were measured at an angle of ca. $45^{\circ}$ to the interface of the spectrometer. Fig. A1b shows the average of 5 measurements for the DFP and 4 measurements for the ZZS, as well as the reflectance of these structures relative to the estimated spectral radiance of a diffuse white standard, based on the attenuation coefficients explained above. This procedure lasted $14 \mathrm{~min}$. Although clove oil anaesthesia tends to reduce the spectral reflectance of specimens (Gray et al. 2011), this effect was not obvious in the rainbow wrasse. Nevertheless, it is likely that natural reflectance is stronger than the measurements shown here. The specimen was released at the site of capture upon recovery from anaesthesia.

Laboratory measures of C. julis

A different male collected at Punta Revellata in $30 \mathrm{~m}$ depth was measured in the laboratory using an AvaSpec 2048 USB spectrometer (Avantes) and a KL 2500 cold light source (Schott) for illumination (Fig. A1c shows the average of 10 reflectance measurements per colour patch [ZZS or DFP]; Fig. A1d shows the spectrum of the cold light source). A Spectralon $99 \%$ diffuse white standard was used as a reference for the measurements. The specimen was anaesthetized with a mixture of clove oil, ethanol and seawater prior to measurements. No darkening of body colouration was observed (see above). Following measurements (lasting $<2 \mathrm{~min}$ ), the specimen was put into a tank with increased flow of seawater to aid recovery from anaesthesia, and was subsequently released near the field site at Punta Revellata.

\section{Behavioural experiment}

\section{General setup}

The behavioural experiment was split into 6 successive runs, with a duration of $5 \mathrm{~d}$ per run. For each run, we established 4 social groups consisting of 1 resident male ( $\mathrm{TL} \pm \mathrm{SE}=150 \pm 13 \mathrm{~mm}$ ) and 2 females $(109 \pm 18 \mathrm{~mm})$ in white fibreglass aquaria (dimensions: 3 tanks of $125 \times 55 \times 58 \mathrm{~cm}$ and 1 tank of $100 \times$
$50 \times 50 \mathrm{~cm}$ ) connected to a flow-through seawater system at ambient water temperature (approx. $24^{\circ} \mathrm{C}$ ). Each aquarium contained a ground covering layer of gravel and sand, 3 algae-overgrown rocks and 2 PVC tubes $(25 \times 6 \mathrm{~cm})$ for shelter. We caught 2 extra males per run $(141 \pm 11 \mathrm{~mm})$ that were housed individually in $60 \times 30 \times 30 \mathrm{~cm}$ flow-through aquaria, each containing some sand and a PVC tube. These males were used as intruders during the experiments. Total sample size was 24 resident males, 12 intruder males and 48 females. Visual interactions between fish of different aquaria were prevented by black curtains. Before and after behavioural observations, fish were kept under room light conditions (fluorescent bulbs) at a 14:10 $\mathrm{h} \mathrm{L}: \mathrm{D}$ photoperiod (starting $1 \mathrm{~h}$ after sunrise), and fed 4 times daily (07:30, 09:00, 12:00 and 18:00 h) with a mixture of fish flakes (TetraMin Naturfutter, Tetra), dried crustaceans (Sawa Meisterklasse) and dried bread.

\section{Light treatments}

RGB LED-floodlights (LED PAR 64, $20 \times 31$ W) were mounted $55 \mathrm{~cm}$ above experimental tanks. The illuminated area focused on the Plexiglas cylinder (Fig. 1b) placed within tanks, but did not totally exclude the rest of the tanks, i.e. the residents' shelter opposite of the cylinder. During the experiment, the fluorescent bulbs of the room lights were turned off. The following 4 different light treatments were used during the experiment (Fig. 2):

- R1, R3 and R5: 3 different mixtures of blue $\left(\lambda_{\max }=\right.$ $454 \mathrm{~nm})$ and red $\left(\lambda_{\max }=628 \mathrm{~nm}\right)$ light, with an increase in intensity of red from R1 to R5, while the same intensity was used for blue light (Fig. 2b-d). In these treatments, residents should be capable of discriminating the long wavelength colours of the DFP and the ZZS from the colours of shorter wavelength (i.e. the remaining body colouration). If the (absolute or relative) intensity of red is important in mediating aggressive behaviour, we would expect an increase in aggression by residents towards intruders with increasing intensity of red from R1 to R5.

- R0: blue ( $\lambda_{\max }=454 \mathrm{~nm}$ ) light only (Fig. 2a). This treatment tested for the importance of the orange/red hue versus the importance of some other feature of the colour patch itself (e.g. shape, size). If aggression by residents towards intruders is triggered by the hue of the DFP and the ZZS, we would expect less aggression in the R0 treatment compared to R1, R3, and R5, simply because the illumination did not contain orange/red wavelengths. If, however, some 


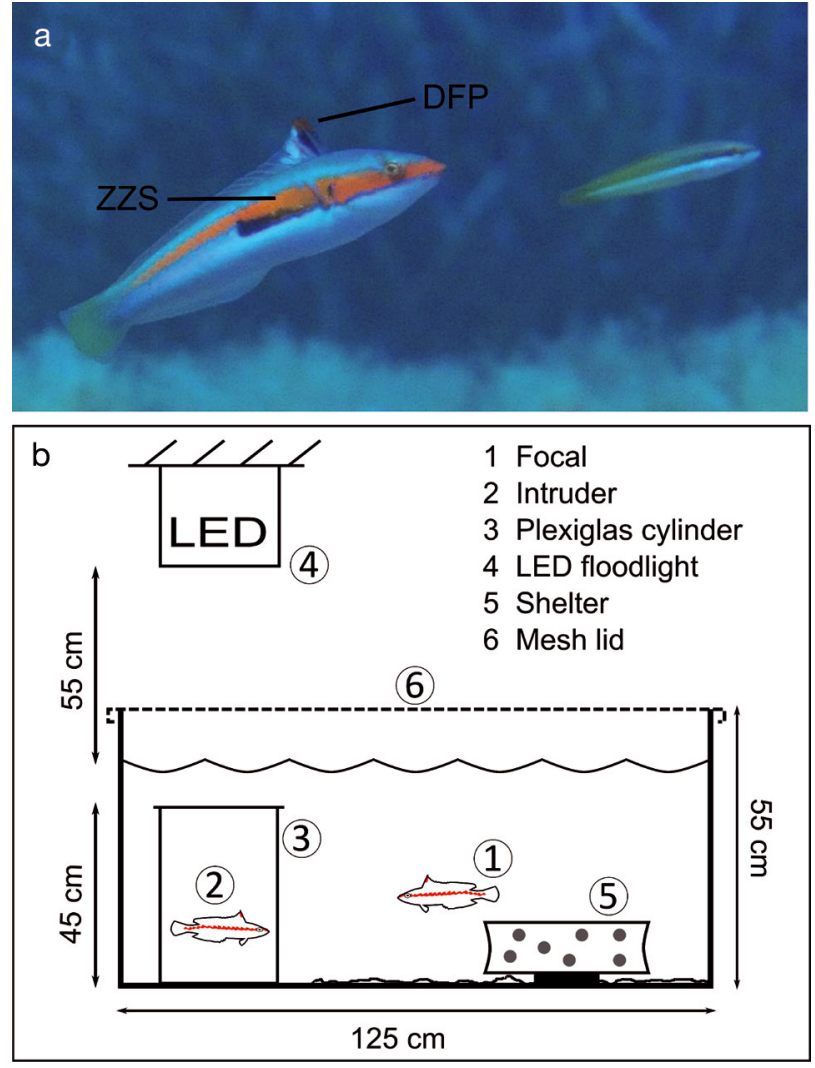

Fig. 1. Study species and experimental setup. (a) Male Coris julis (left) displaying to a female (right) at a depth of $12 \mathrm{~m}$. Note the red dorsal fin patch (DFP) and the orange zigzag stripe (ZZS) of the male. Picture was taken with a Nikon D300 in a Hugyfoot housing equipped with a LEE filter (flame red). (b) Schematic drawing of the experimental setup. Note that the LED floodlight was positioned directly above the Plexiglas cylinder containing the intruder fish

other feature of the ZZS or DFP was important in agonistic interactions, no difference in aggression would be expected between R0 and the remaining treatments since those features should still be available to the fish in R0, if we assume that they possess at least 2 visual pigments sensitive to short and long wavelength light (Barry \& Hawryshyn 1999).

According to our hypothesis, we predicted that a resident's behaviour would be mediated by the presence as well as the quantity of red light relative to a constant background illumination. Our setup did not allow us to differentiate whether changes in behaviour were mediated by long wavelength light only or by a change in total light intensity, yet we considered the latter negligible as changes in overall intensity of a treatment are only manifested by long wavelength light. The intensity of the blue light component of our treatments remained unchanged.

\section{Acclimation and test phase}

During the first $4 \mathrm{~d}$ of each run, fish of the 4 social groups were accustomed to the presence of the observer, the different light conditions used during test trials (see below), and an empty Plexiglas cylinder $(45 \times 24 \mathrm{~cm}$, light transmittance 92\%; Evonik Röhm). Pilot observations revealed that males in the field strongly attack (i.e. biting attempts) intruders placed within the Plexiglas cylinder.

Test trials with the different light treatments were conducted on the last day of a run, (i.e. Day 5 after capture). During test trials, each resident (1 per social group; 4 in total per run) was subsequently presented with each of the 2 intruder males for $2 \mathrm{~min}$, in each of the 4 different light conditions - resulting in 8 test trials per resident ( 2 per light condition, each with a different intruder). The transition between light treatments was $30 \mathrm{~s}$ long, in which no behaviours were recorded.

To exclude order effects, we randomized the sequence of presentation of the 2 intruders among the 4 residents per run. We also randomized the sequence of light treatments among residents under which an intruder was presented. Before placing an intruder into the Plexiglas cylinder, LED lights were set to $50 \%$ intensity of treatment R0 (see below). Following each run, all fish were removed, tanks were cleaned, and water was exchanged.

\section{Behavioural recordings}

During test trails, fish were observed through a slit in a black curtain, and the following behaviours were recorded: (1) the occurrence of an attack versus no attack by residents. For each light treatment, we noted whether residents did or did not direct an attack towards the intruder (binary variable with attack $=1$, no attack $=0$ ). An attack was defined as the resident shooting forward and touching the plastic cylinder with its mouth; (2) the number of attacks by residents towards intruders, by counting how often residents attacked an intruder per light treatment; (3) the occurrence of dorsal fin display by an intruder, by recording for each light treatment whether an intruder did or did not display its dorsal fin while being attacked (binary variable with display $=1$, no display $=0$ ). None of the fish tested showed any form of physical injury or signs of physical trauma or stress at the end of an experimental run. 


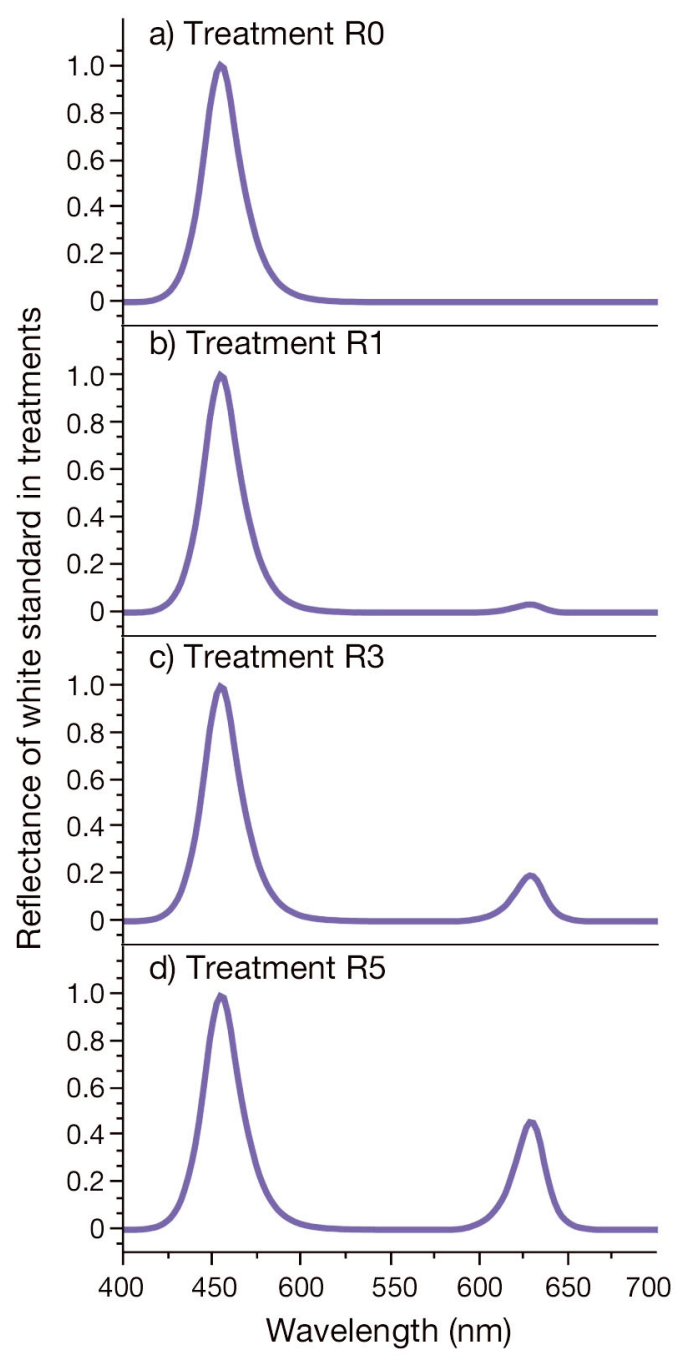

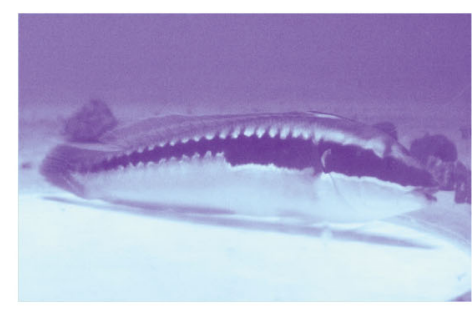
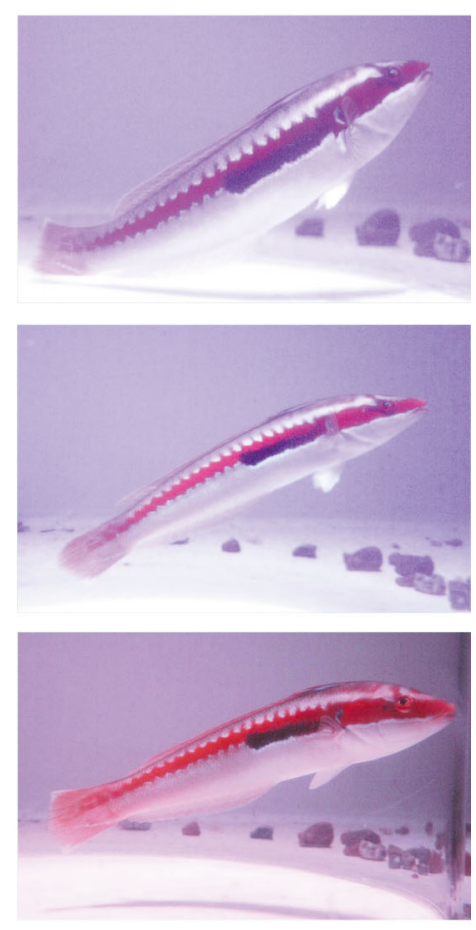

Fig. 2. Light treatments used in the behavioural experiment. Reflectance spectra of a white standard (graphs) and the appearance of the same male Coris julis intruder to a human observer in the 4 experimental light treatments (photographs). (a) Blue light treatment R0. (b-d) Light treatments R1, R3 and R5, with increasing intensity of red light from R1 to R5 while the intensity of blue light remained constant. Spectra were measured using the same white standard (Spectralon diffuse white, Labsphere) placed in one of the aquaria filled with sea water (water depth approx. $50 \mathrm{~cm}$; see Fig. 1b). Measurements plotted on the $y$ axes were normalized by setting the highest measured reflectance equal to 1

\section{Colour patch dimensions}

Following each test run and within $1 \mathrm{~h}$ of the run, we measured the following parameters for each of the 4 residents and 2 intruders: (1) TL, (2) body area, (3) ZZS area, and (4) DFP area. Measurements were taken from both body sides, and the mean per individual fish was used for further analyses. Fish were anaesthetized with a mixture of clove oil, ethanol, and sea water $(1: 1: 100)$ and gently placed onto a scanner (Brother DCP-385C, image resolution $600 \times$ 600 dpi). A layer of silicone was glued along the edges of the scanner, such that fish could be maintained in seawater while being measured. A ruler was taped to the glass plate to scale measurements. During scans (each lasting $30 \mathrm{~s}$ ), the dorsal fin was erected with a blunt dissection needle. Immediately thereafter, fish were put into a tank with increased flow of seawater to aid with recovery from the anaes- thesia. All fish were released near the site of capture. All images where analyzed with FIJI v1.43 (Schindelin et al. 2012). The average dimensions of these areas for all fish were $5.84 \pm 0.86 \mathrm{~cm}^{2}$ for the DFP, and $0.33 \pm 0.1 \mathrm{~cm}^{2}$ for the $\mathrm{ZZS}$.

\section{Statistical analysis}

To test our first hypothesis (i.e. that residents are more likely to attack in the presence versus the absence of red light), we fitted a generalized linear mixed model (GLMM) with a binomial error distribution and a logit-link function using the lme4 package (Bates \& Maechler 2010) in R 2.15.2 (R Development Core Team 2012). The response variable was 'attack' (binary variable with yes $=1$, no $=0$ ) and the fixed effect was 'light treatment' with R0 (i.e. blue light only) set as the reference treatment, such that all 
other treatments (R1，R3，R5) were compared to R0. Experimental run, resident ID and intruder ID were included as random intercepts, with the latter 2 accounting for non-independent measures (multiple measures per resident across the different light treatments per run, and multiple use of 2 intruders among the 4 social groups per run). Covariates were the differences in the DFP and the ZZS area between residents and intruders (see Hypothesis 2: that the likelihood of aggression depends on the relative difference in signal size of the DFP and the ZZS between residents and intruders). There was no correlation between the DFP and ZZS in both residents (pairwise correlation: $\mathrm{n}=24, \mathrm{r}=-0.14, \mathrm{p}=0.50$ ) and intruders (pairwise correlation: $\mathrm{n}=12, \mathrm{r}=-0.12, \mathrm{p}=$ 0.71 ). There was also no correlation between the DFP and body size both in intruders (pairwise correlation: $\mathrm{n}=12, \mathrm{r}=0.34, \mathrm{p}=0.27$ ) and residents (pairwise correlation: $\mathrm{n}=24, \mathrm{r}=0.17, \mathrm{p}=0.42$ ). While the ZZS was independent of body size in intruders (pairwise correlation: $\mathrm{n}=12, \mathrm{r}=0.13, \mathrm{p}=0.68$ ), we found a positive correlation between ZZS and body size in residents (pairwise correlation: $\mathrm{n}=24, \mathrm{r}=0.74, \mathrm{p}<0.001$ ). The latter remained significant $(p=0.006)$ after applying the false discovery rate control to correct for multiple testing (Benjamini \& Hochberg 1995, Verhoeven et al. 2005).

Covariates were calculated by first deriving residuals from linear regression analysis between the DFP and the ZZS and body size for both residents and intruders separately. We then subtracted the residuals of intruders from those of residents for the DFP and ZZS. To account for an intruder's behaviour, we also included dorsal fin display by intruders (binary variable with dorsal fin displayed $=1$, dorsal fin not displayed $=0$ ) as well as the interaction term dorsal fin display by intruders $\times$ light treatment into the model. In case of a non-significant interaction term, we performed model selection based on Bayesian information criterion (BIC) scores, with a difference of $\geq 2$ indicating a better model fit (Zuur et al. 2009). Because the likelihood for an attack by residents was found to be significantly affected by an intruder's behaviour (Table 1a), we fitted a second GLMM to test whether the likelihood for dorsal fin display by intruders (response variable with dorsal fin displayed $=1$, dorsal fin not displayed $=0$ ) differed across treatments using a binary error distribution and a logit-link function. Random intercepts were resident ID, intruder ID, and experimental run.

To address our third hypothesis (i.e. that the number of attacks by residents increases with increasing intensity of red in the light environment), we quanti-
Table 1. Generalized linear mixed model (GLMM) results for the effect of light treatment on (a) the likelihood of an attack by resident Coris julis towards intruders in treatments R1, R3, and R5 compared to R0, (b) the likelihood of dorsal fin display by intruders towards residents in treatments R1, R3, and R5 compared to R0, (c) the number of attacks by residents towards intruders in treatments R3 and R5 compared to R1. Significant values $(p<0.05)$ in bold

\begin{tabular}{|c|c|c|c|c|}
\hline & Estimate & SE & $Z$ & $\mathrm{p}$ \\
\hline \multicolumn{5}{|c|}{ (a) Likelihood of attacks by residents } \\
\hline Intercept & -2.85 & 0.67 & -4.20 & $<0.001$ \\
\hline $\mathrm{R} 1$ & 1.06 & 0.59 & 1.80 & 0.072 \\
\hline R3 & 0.51 & 0.60 & 0.85 & 0.394 \\
\hline R5 & 1.50 & 0.59 & 2.55 & 0.011 \\
\hline Intruder fin display & 1.49 & 0.54 & 2.79 & 0.005 \\
\hline DFP area & -0.67 & 0.49 & -1.38 & 0.168 \\
\hline ZZS area & 0.27 & 0.45 & 0.61 & 0.543 \\
\hline \multicolumn{5}{|c|}{ (b) Likelihood of dorsal fin display by intruders } \\
\hline Intercept & -1.44 & 0.54 & -2.68 & 0.007 \\
\hline R1 & 1.05 & 0.50 & 2.09 & 0.037 \\
\hline R3 & 0.15 & 0.53 & 0.28 & 0.780 \\
\hline R5 & -0.16 & 0.55 & -0.29 & 0.775 \\
\hline \multicolumn{5}{|c|}{ (c) Number of attacks by residents } \\
\hline Intercept & -1.93 & 0.52 & -3.74 & $<0.001$ \\
\hline R3 & -0.68 & 0.43 & -1.58 & 0.115 \\
\hline R5 & -0.16 & 0.41 & -0.40 & 0.691 \\
\hline Intruder fin display & 1.73 & 0.41 & 4.24 & $<0.001$ \\
\hline DFP area & -5.45 & 3.08 & -1.77 & 0.077 \\
\hline ZZS area & 0.15 & 0.42 & 0.36 & 0.723 \\
\hline
\end{tabular}

fied differences in the number of attacks between R1 (set as reference treatment, R0 excluded) and the remaining treatments R3 and R5 using a GLMM with a Poisson error distribution and a log-link function corrected for overdispersion (Zuur et al. 2009). Otherwise, model parameters were the same as in the first GLMM.

\section{RESULTS}

Residents were significantly more likely to attack intruders in the R5 treatment compared to the R0 treatment ( $p=0.011, Z=2.55)$, while no such effect was detected for the R1 and R3 treatments (Fig. 3a, Table 1a). There was no difference in the number of attacks by residents in treatments R3 and R5 relative to R1 (Tables 1c \& 2). The presence of attacks by residents and the number of attacks by residents were significantly associated with an increased display of an intruder's dorsal fin (presence of attacks: $p=$ $0.005, Z=2.79$; number of attacks: $\mathrm{p}<0.001, Z=4.24$; Table $1 \mathrm{a}, \mathrm{c})$. Specifically, intruders displayed their dorsal fin in all 4 treatments (Fig. 3b), with a significantly higher likelihood of dorsal fin display in the R1 


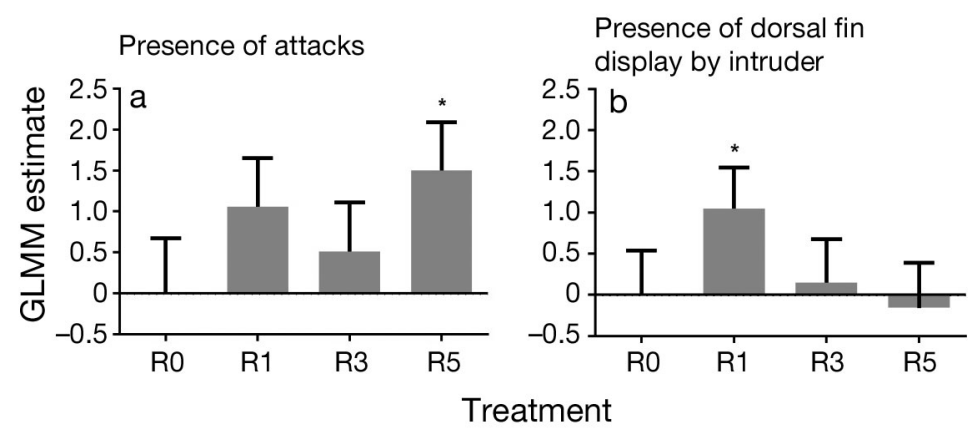

Fig. 3. Presence of (a) attacks and (b) dorsal fin display by Coris julis intruders during encounters across the different light treatments. Error bars indicate $\mathrm{SE}_{\text {; }}$ asterisks indicate significant differences from $\mathrm{R} 0$ treatment $(p<0.05)$

Table 2. Mean number of attacks per resident Coris julis towards the intruder male

\begin{tabular}{|lcc|}
\hline Treatment & Mean & SD \\
\hline R0 & 1.04 & 2.62 \\
R1 & 2.58 & 5.63 \\
R3 & 1.06 & 2.52 \\
R5 & 0.88 & 1.33 \\
\hline
\end{tabular}

treatment compared to the R0 treatment $(\mathrm{p}=0.037$, $Z=2.09$; Table $1 \mathrm{~b}$ ), while no such difference was found between R0 and treatments R3 and R5. Neither the DFP nor the ZZS areas were found to affect a resident's aggressive behaviour (Table 1a,c).

\section{DISCUSSION}

Here, we provide experimental support for the signalling function of long wavelength colours during aggressive male-male interactions in the Mediterranean rainbow wrasse Coris julis. We found an increased likelihood for attacks by residents towards intruders when light settings allowed for discrimination between long and short wavelength colours (i.e. a mixture of red and blue light in treatment R5) compared to conditions in which no such discrimination was possible (i.e. blue light in treatment R0). This means that long wavelength light increases aggressive interactions in males of this species.

Our results are concordant to previous work in three-spined sticklebacks Gasterosteus aculeatus, with males being significantly more aggressive towards conspecific intruders (Baube 1997) and more attractive to females (Rick \& Bakker 2008) in light environments in which short wavelength colours could be distinguished from colours of longer wave- lengths, while monochromatic treatments showed no significant effects.

While we also found a trend towards an increased likelihood for attacks by residents in the R1 treatment, this coincided with an increased dorsal fin display by intruders. However, no such effect was observed in the R5 treatment, suggesting that the increased likelihood for an aggressive response in R5 was not solely triggered by an increase in the dorsal fin display by intruders.

We found no evidence for our second hypothesis that the area of the red DFP and/or of the orange ZZS mediates malemale aggression in $C$. julis. Size, as well as colouration of a highly visible signal used in a territorial interaction (i.e. a badge) has previously been shown to play an important role in aggressive interactions between male red-collared widowbirds (Pryke et al. 2001, 2002) and juvenile salmon $\left(\mathrm{O}^{\prime}\right.$ Connor et al. 1999). In the widowbird study, males with smaller collars were engaged longer in fights with more attacks, while the salmon showed darker colouration to signal subordinance. In contrast, we observed a trend for male residents with relatively smaller DFP or DFP sizes to attack more often, however, the outcome was non-significant. Contestants in our experiment were not matched for either DFP or ZZS size or colouration, and aggressive interactions could have been prevented by these differences. Future experiments with males of this species should consider matching for these factors, since game theory (Riechert 1998) predicts a higher likelihood for the escalation of a fight if contestants' signals are matched for size.

Contrary to our third prediction, we found no increase in the number of attacks with an increasing intensity of red light in treatments R1 to R5. In contrast to Baube's (1997) findings on the importance of the red colouration of sticklebacks in mate choice, our results suggest that the strength of aggression in Mediterranean rainbow wrasses is primarily mediated by the presence rather than the intensity of red light. This could be due to a mismatch of the peak emittance of the red LEDs used in the light treatments (around $628 \mathrm{~nm}$ ), and the (yet unknown) spectral sensitivity of our study species, which might be shifted towards shorter wavelengths around $570 \mathrm{~nm}$, as in other labrids (Barry \& Hawryshyn 1999, Marshall et al. 2006).

Importantly, while these results were obtained under artificial lighting conditions, they are unlikely 
to represent a mere laboratory effect. This is because spectrometric field measures suggest that long wavelength colours are, in theory, still detectable at $21 \mathrm{~m}$ depth. Moreover, the peak of the ZZS and the secondary peak of the DFP at around $575 \mathrm{~nm}$ coincide well with the longest $\lambda_{\max }$ values $(580 \mathrm{~nm}$ ) for photoreceptors measured in reef fish (Marshall et al. 2006) and the spectral sensitivity of another labrid (Barry \& Hawryshyn 1999), so that visual detection of the putative colour signals is theoretically possible. Although our results show that the light environment mediated the likelihood of an attack in C. julis, the total number of attacks was low. We speculate that this might be due to 3 reasons. First, $4 \mathrm{~d}$ of acclimation may have been insufficient for residents to display full natural territorial behaviour in an aquarium. Also, and in contrast to our expectations, the presence of females in the experimental tanks might have led to a reduction in aggression - as documented in the Siamese fighting fish Betta splendens (Doutrelant et al. 2001). Although females generally did not interact with males during the trials, we cannot exclude their influence. Second, aggressive interactions may have been settled prior to the escalation of a fight, with residents assessing the aggressive potential of intruders (e.g. by the display of the DFP), thereby avoiding costly fights with opponents that posed no threat or were clearly superior. Thirdly, a manipulation of the visibility of certain signals or colour patches that might be important for species recognition has been shown to influence levels of aggression between intruder and resident males (Siebeck et al. 2010), and was not investigated in this study. Therefore, a breakdown of this important process in our experiment might have led to a lower level of aggression compared to the field results.

Taken together, this study provides experimental support for the hypothesis of long wavelength colours mediating intraspecific behavioural interactions in marine fish. Specifically, we found that aggressive interactions of male $C$. julis increased with light conditions that allowed for discriminating long wavelength colours from those of shorter wavelength, as well as on the display of a red coloured patch on the dorsal fin. These results call for more work investigating the functional importance of long wavelength colours in the sea.

Acknowledgements. We thank the staff of STARESO for logistical support, Ines Häderer for help with field work and assistance during the behavioural experiments, and Pierre Lejeune for discussion. This work was funded by a PROMOS Research Grant provided by the Deutscher Akademischer Austauschdienst (German Academic Exchange Service).

\section{LITERATURE CITED}

Andersson M, Iwasa Y (1996) Sexual selection. Trends Ecol Evol 11:53-58

> Bakker TCM, Mundwiler B (1994) Female mate choice and male red coloration in a natural three-spined stickleback (Gasterosteus aculeatus) population. Behav Ecol 5:74-80

Bakker T, Sevenster P (1983) Determinants of dominance in male sticklebacks (Gasterosteus aculeatus L.). Behaviour 86:55-71

Barry KL, Hawryshyn CW (1999) Spectral sensitivity of the Hawaiian saddle wrasse, Thalassoma duperrey, and implications for visually mediated behaviour on coral reefs. Environ Biol Fishes 56:429-442

> Basolo AL (1990) Female preference for male sword length in the green swordtail, Xiphophorus helleri (Pisces: Poeciliidae). Anim Behav 40:332-338

Bates D, Maechler M (2010) lme4: linear mixed-effects models using S4 classes. http://CRAN.R-project.org/ package $=$ lme 4

Baube CL (1997) Manipulations of signalling environment affect male competitive success in three-spined sticklebacks. Anim Behav 53:819-833

Benjamini Y, Hochberg Y (1995) Controlling the false discovery rate: a practical and powerful approach to multiple testing. J R Stat Soc B 57:289-300

Benson KE, Basolo AL (2006) Male-male competition and the sword in male swordtails, Xiphophorus helleri. Anim Behav 71:129-134

$>$ Douglas RH, Mullineaux CW, Partridge JC (2000) Longwave sensitivity in deep-sea stomiid dragonfish with far-red bioluminescence: evidence for a dietary origin of the chlorophyll-derived retinal photosensitizer of Malacosteus niger. Philos Trans R Soc Lond B Biol Sci 355: 1269-1272

> Doutrelant C, McGregor PK, Oliveira RF (2001) The effect of an audience on intrasexual communication in male Siamese fighting fish, Betta splendens. Behav Ecol 12: 283-286

> Duchac B, Huber F, Müller H, Senn D (1982) Mating behavior and cytogenetical aspects of sex-inversion in the fish Coris julis L. (Labridae, Teleostii). Cell Mol Life Sci 38: 809-811

Franck D, Müller A, Rogmann N (2003) A colour and size dimorphism in the green swordtail (population Jalapa): female mate choice, male-male competition, and male mating strategies. Acta Ethol 5:75-79

> Gray SM, Hart FL, Tremblay MEM, Lisney TJ, Hawryshyn CW (2011) The effects of handling time, ambient light, and anaesthetic method, on the standardized measurement of fish colouration. Can J Fish Aquat Sci 68: 330-342

Jerlov NG (1968) Optical oceanography. Elsevier, Amsterdam Kondrashev SL (2008) Long-wave sensitivity in the masked greenling (Hexagrammos octogrammus), a shallowwater marine fish. Vision Res 48:2269-2274

Lejeune P (1987) The effect of local stock density on social behavior and sex change in the Mediterranean labrid Coris julis. Environ Biol Fishes 18:135-141

Lythgoe JN (1979) The ecology of vision. Oxford University Press, New York, NY

Marshall NJ, Jennings K, McFarland WN, Loew ER, Losey GS, Montgomery WL (2003) Visual biology of Hawaiian coral reef fishes. III. Environmental light and an integrated approach to the ecology of reef fish vision. Copeia 
2003:467-480

Marshall NJ, Vorobyev M, Siebeck UE (2006) What does a reef fish see when it sees a reef fish? Eating 'Nemo@'. In: Ladich F, Collin SP, Moller P, Kapoor BG (eds) Communication in fishes, Vol 2. Science Publisher, Enfield, CT, p 393-422

Michiels NK, Anthes N, Hart NS, Herler J and others (2008) Red fluorescence in reef fish: a novel signalling mechanism? BMC Ecol 8:16

Milinski M, Bakker TCM (1990) Female sticklebacks use male coloration in mate choice and hence avoid parasitized males. Nature 344:330-333

O'Connor KI, Metcalfe NB, Taylor AC (1999) Does darkening signal submission in territorial contests between juvenile Atlantic salmon, Salmo salar? Anim Behav 58: 1269-1276

Pryke SR, Lawes MJ, Andersson S (2001) Agonistic carotenoid signalling in male red-collared widowbirds: aggression related to the colour signal of both the territory owner and model intruder. Anim Behav 62:695-704

Pryke SR, Andersson S, Lawes MJ, Piper SE (2002) Carotenoid status signaling in captive and wild red-collared widowbirds: independent effects of badge size and color. Behav Ecol 13:622-631

R Development Core Team (2012) R: a language and environment for statistical computing. R Foundation for Statistical Computing, Vienna

Rick IP, Bakker TCM (2008) Color signaling in conspicuous red sticklebacks: Do ultraviolet signals surpass others? BMC Evol Biol 8:189

Riechert SE (1998) Game theory and animal contests. In: Dugatkin LA, Reeve HK (eds) Game theory and animal behavior. Oxford University Press, Oxford, p 64-93

Schindelin J, Arganda-Carreras I, Frise E, Kaynig V and others (2012) Fiji: an open-source platform for biologicalimage analysis. Nat Methods 9:676-682

Seehausen O, van Alphen JJM (1998) The effect of male coloration on female mate choice in closely related Lake Victoria cichlids (Haplochromis nyererei complex). Behav Ecol Sociobiol 42:1-8

> Seehausen O, van Alphen JJM, Witte F (1997) Cichlid fish diversity threatened by eutrophication that curbs sexual selection. Science 227:1808-1811

- Siebeck UE (2004) Communication in coral reef fish: the role of ultraviolet colour patterns in damselfish territorial behaviour. Anim Behav 68:273-282

Siebeck UE, Parker AN, Sprenger D, Mäthger LM, Wallis G (2010) A species of reef fish that uses ultraviolet patterns for covert face recognition. Curr Biol 20:407-410

Sparks JS, Schelly RC, Smith WL, Davis MP, Tchernov D, Pieribone VA, Gruber DF (2014) The covert world of fish biofluorescence: a phylogenetically widespread and phenotypically variable phenomenon. PLoS ONE 9: e83259

> Stacey PB, Chiszar D (1978) Body color pattern and the aggressive behavior of male pumpkinseed sunfish (Lepomis gibbosus) during the reproductive season. Behaviour 64:271-297

> Verhoeven KJF, Simonsen KL, McIntyre LM (2005) Implementing false discovery rate control: increasing your power. Oikos 108:643-647

Zuur A, Ieno E, Walker N, Saveliev A, Smith G (2009) Mixed effects models and extensions in ecology with $\mathrm{R}$. Springer, New York, NY 


\section{Appendix.}
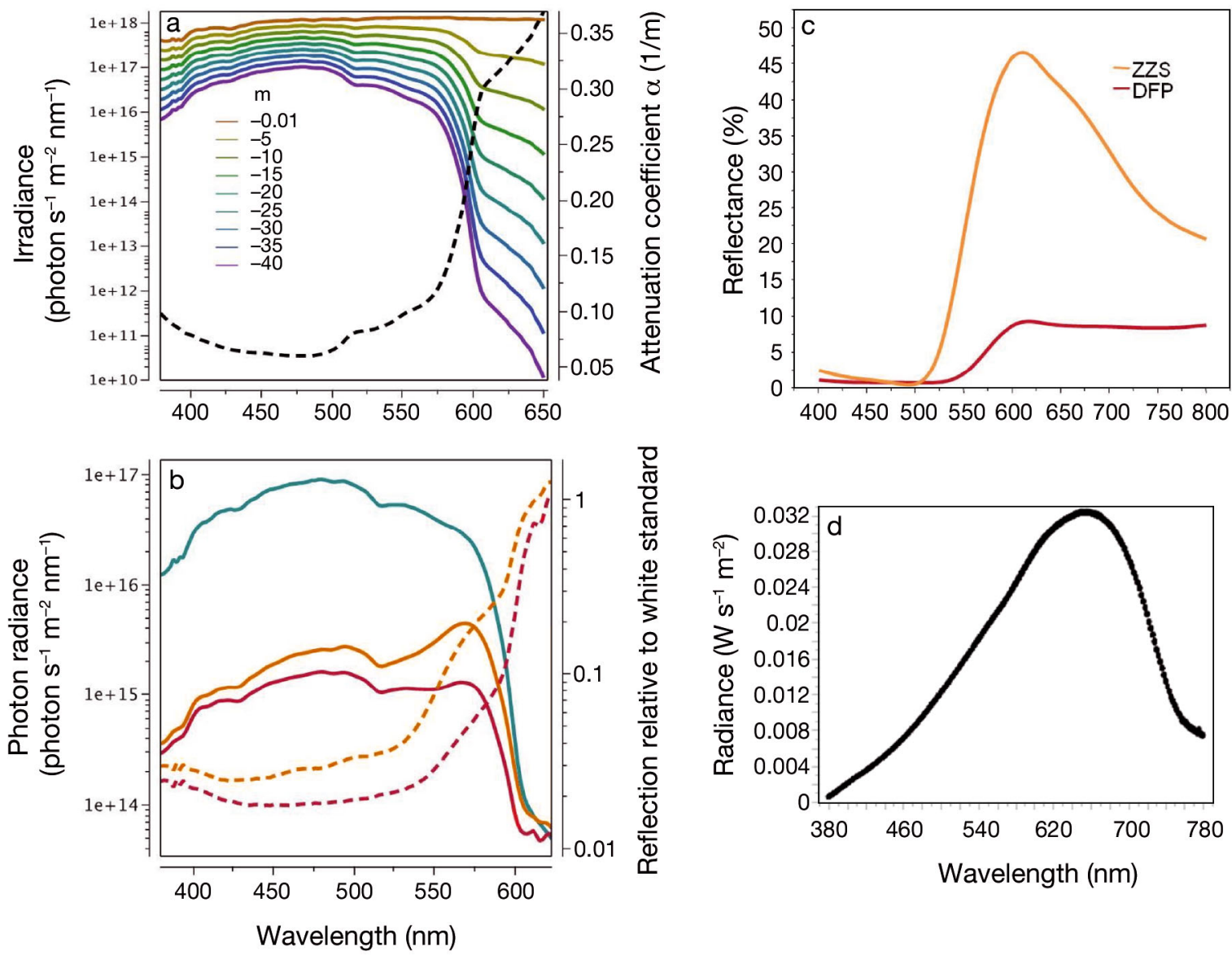

Fig. A1. Spectrometric measurements. (a) Light environment at the Punta Revellata study site. Note the shift towards bluegreen light with increasing depth due to absorbance of long wavelengths as indicated by a higher attenuation coefficient, $\alpha$ (dashed line). (b) Spectral measurements of a male Coris julis in the field at a depth of $23 \mathrm{~m}$. Solid lines show absolute radiance blue $=$ Spectralon white standard, orange = zigzag stripe $($ ZZS), red $=$ dorsal fin patch $(D F P)$. Dashed lines show the reflection of the colour patches relative to the white standard: orange $=$ ZZS, red $=$ DFP. The ZZS shows peak radiance at around $575 \mathrm{~nm}$ at this depth, and a secondary peak at this wavelength is visible for the DFP. (c) Reflectance of male $C$. julis colour patches illuminated with a cold light source relative to a Spectralon white standard. Peak reflectance of both the ZZS and the DFP is at ca. $610 \mathrm{~nm}$. (d) Emittance of the cold light source used for the laboratory reflectance measurements of $C$. julis colour patches. The radiance was measured by measuring directly into the light source using the photospectrometer PR-670 mentioned in 'Materials and methods: Spectrometric measures'

Editorial responsibility: Nicholas Tolimieri, Seattle, Washington, USA
Submitted: September 13, 2013; Accepted: February 7, 2014 Proofs received from author(s): April 16, 2014 